\title{
THE NILPOTENCY OF SOME GROUPS WITH ALL SUBGROUPS SUBNORMAL
}

\author{
Leonid A. Kurdachenko and Howard Smith
}

\begin{abstract}
Let $G$ be a group with all subgroups subnormal. A normal subgroup $N$ of $G$ is said to be $G$-minimax if it has a finite $G$-invariant series whose factors are abelian and satisfy either $\max -G$ or min$G$. It is proved that if the normal closure of every element of $G$ is $G$-minimax then $G$ is nilpotent and the normal closure of every element is minimax. Further results of this type are also obtained.
\end{abstract}

\section{Introduction}

Let $G$ be a group with all subgroups subnormal. If the normal closure in $G$ of every element is finitely generated then $G$ is nilpotent $[\mathbf{1 5}$, Theorem 1]. We deal in the present paper with the case where every normal closure is minimax. Let us note at the outset that there is no corresponding result for the case where normal closures have finite (Prüfer) rank; indeed, an example in $[\mathbf{1 2}]$ shows that even if $G$ itself has finite rank then it need not be nilpotent.

Using standard notation, we denote by $\mathfrak{S}_{2}$ the class of soluble minimax groups; thus a group $G$ belongs to $\mathfrak{S}_{2}$ if it has a finite normal series the factors of which are abelian and satisfy either max or min. Since every group with all subgroups subnormal is known to be soluble [9], the hypothesis that the normal closure of every element belong to $\mathfrak{S}_{2}$ is less restrictive than it might at first appear. In fact, we shall establish a somewhat stronger result than that hinted at above. A normal subgroup $N$ of a group $G$ is said to be $G$-minimax if it has a finite $G$ invariant series of subgroups the factors of which are abelian and satisfy either $\max -G$ or min- $G$. Our first result is as follows. 
Theorem A. Let $G$ be a group with all subgroups subnormal. If $\langle x\rangle^{G}$ is $G$-minimax for all $x$ in $G$ then

(i) $G$ is nilpotent and

(ii) $\langle x\rangle^{G}$ is minimax for all $x$ in $G$.

A group $G$ belongs to the class $\mathfrak{S}_{1}$ if it has a finite normal abelian series the factors of which are torsion-free of finite rank or Chernikov groups. (Thus $\mathfrak{S}_{2} \subseteq \mathfrak{S}_{1}$. Additional care needs to be taken when dealing with the class $\mathfrak{S}_{1}$ since it is not closed under forming quotients, as may be seen by considering the additive group of rationals.)

Theorem B. Let $G$ be a group with all subgroups subnormal and suppose that $\langle x\rangle^{G} \in \mathfrak{S}_{1}$ for all $x$ in $G$. Then $G$ is nilpotent.

Using Theorem B we are able to deduce a further result. For a class $\mathfrak{X}$ of groups, a group $G$ is said to be an $\mathfrak{X} C$-group if $G / C_{G}\left(x^{G}\right)$ belongs to $\mathfrak{X}$ for all $x$ in $G$. In the case where $\mathfrak{X}=\mathfrak{S}_{2}$ we have the class of groups with "minimax conjugacy classes". Now, by Theorem 2 of [6], if $G \in \mathfrak{S}_{2} C$ then $\langle x\rangle^{G} \in \mathfrak{S}_{2}$ for all $x \in G$, and we have the following consequence of Theorem B.

Corollary. Let $G$ be a group with all subgroups subnormal and suppose that $G$ has minimax conjugacy classes. Then $G$ is nilpotent.

There are no doubt several possible generalisations of Theorem B; we content ourselves with establishing a result that has both Theorem A(i) and Theorem B as special cases.

Theorem C. Let $G$ be a group with all subgroups subnormal and suppose that, for each $x$ in $G,\langle x\rangle^{G}$ has a finite $G$-invariant series each of whose factors is either $G$-minimax or torsion-free abelian of finite rank. Then $G$ is nilpotent.

\section{2. $\max -G$ and min- $G$ subgroups of locally nilpotent groups}

We begin with a couple of definitions. Let $G$ be a group, $H$ an infinite normal subgroup of $G$. Then $H$ is $G$-quasifinite if every proper $G$-invariant subgroup $N$ of $H$ is finite and $H$ is the join of all such subgroups $N$, while $H$ is $G$-just infinite if every nontrivial $G$-invariant subgroup $N$ of $H$ has finite index in $H$ and the intersection of all such $N$ is trivial. For a group $G$, just infinite $\mathbf{Z} G$-modules were first studied in [11] and [5], quasifinite $\mathbf{Z} G$-modules in [16]. For further references the reader is invited to consult the survey [4]. Our first requirement is as follows. 
Lemma 2.1. Let $G$ be a locally nilpotent group, $H$ an infinite normal subgroup of $G$. If $H$ satisfies min- $G$ then $H$ contains a $G$-invariant subgroup $A$ that is $G$-quasifinite.

Proof: By min- $G$ we see that $H$ certainly contains a $G$-invariant subgroup $A$ minimal with respect to being infinite. If $A$ is not $G$-quasifinite then the join $F$ of all finite $G$-invariant subgroups of $A$ is finite; then $A / F$ is a chief factor of $G$ and therefore finite (of prime order) and we have the contradiction that $A$ is finite.

Now let $G$ and $A$ be as above. If $B$ is a proper $G$-invariant subgroup of $A$ then $B$ is finite and therefore so is $A / C_{A}(B)$, and it follows that $B$ is contained in the centre of $A$; thus $A$ is abelian. Clearly $A$ is a $p$-group for some prime $p$. If the subgroup $C$ generated by all elements of order $p$ in $A$ is infinite then $C=A$; otherwise $C$ is finite and $A$ is Chernikov [10, 25.1] and therefore divisible. Thus we have the following.

Lemma 2.2. Let $G$ be a locally nilpotent group, $A$ a G-quasifinite subgroup of $G$. Then $A$ is abelian and either of exponent $p$ or a divisible Chernikov p-group, where $p$ is a prime.

Next we establish a result that rules out the first of these two possibilities in certain circumstances. Recall that a Baer group is a group in which every cyclic subgroup is subnormal.

Lemma 2.3. Let $G$ be a Baer group, $A$ a $G$-quasifinite subgroup of $G$. If $G / C_{G}(A)$ is hypercentral then $A \leq Z(G)$; in particular $A$ is divisible Chernikov.

Proof: Supposing the result false, choose $z \in G$ with $z C_{G}(A)$ a nontrivial element of $Z\left(G / C_{G}(A)\right)$. By Lemma $2.2 A$ is abelian; from the choice of $z$ it follows easily that both $[A, z]$ and $C_{A}(z)$ are normal in $G$. Further, if $[A, z]<A$ then $[A, z]$ is finite and so $C_{A}(z)$ has finite index in $A$ and hence equals $A$, a contradiction; thus $A=[A, z]$. Write $H=A\langle z\rangle$. Since $G$ is a Baer group, $H$ is nilpotent. But $H^{\prime}=[A,\langle z\rangle]=$ $A=[A, H]=\left[H^{\prime}, H\right]$ and it follows that $H^{\prime}=1$. This again contradicts the choice of $z$ and establishes the result.

We are now able to prove the following.

Lemma 2.4. Let $G$ be a Baer group, $A$ a normal subgroup of $G$ such that $G / C_{G}(A)$ is hypercentral. Suppose that $A$ satisfies min-G. Then $A$ is Chernikov and $A \leq Z_{n}(G)$ for some positive integer $n$. 
Proof: If $A$ is finite the result is clear. Otherwise, we may apply Lemma 2.1 to obtain a $G$-invariant subgroup $B_{1}$ of $A$ that is $G$-quasifinite and hence, by Lemma 2.3, divisible Chernikov and central in $G$. Assuming the statement of the lemma false, repeated application of this argument gives an ascending chain of $G$-quasifinite factors $B_{i} / B_{i-1}$ where, for each $i \geq 1, B_{i} \leq Z_{i}(G)$ and $B_{i} / B_{i-1}$ is divisible Chernikov (interpreting $B_{0}$ as 1$)$. Write $B=\bigcup_{i=1}^{\infty} B_{i}$. Each $B_{i}$ is divisible Chernikov and hence abelian, so that $B$ is abelian but not Chernikov. By min- $G$, some prime component $P$ of $B$ has infinite rank and hence contains an infinite $G$-invariant subgroup $Q\left(=\Omega_{1}(P)\right)$ of exponent $p[\mathbf{1}, 25.1]$. But $Q$ satisfies min- $G$ and so by Lemma 2.1 contains a $G$-invariant subgroup $R$ that is $G$-quasifinite. Lemma 2.3 now gives the contradiction that $R$ is divisible, and the lemma is proved.

We turn now to discussion of normal subgroups satisfying max- $G$, beginning with the counterpart to Lemma 2.1 .

Lemma 2.5. Let $G$ be a locally nilpotent group, $H$ an infinite normal subgroup of $G$. If $H$ satisfies max- $G$ then $H$ contains a $G$-invariant subgroup $A$ such that $H / A$ is $G$-just infinite (that is, $G / A$-just infinite).

Proof: Let $A$ be a $G$-invariant subgroup of $H$ maximal with respect to $H / A$ being infinite and let $B$ denote the intersection of all $G$-invariant subgroups $N$ of $H$ that properly contain $A$. If $B>A$ then $H / B$ is finite and $B / A$ is a chief factor of $G$ and therefore finite. This gives the contradiction that $H / A$ is finite.

Lemma 2.6. Let $G$ be a locally nilpotent group, $A$ a G-just infinite subgroup. Then either $A$ is torsion-free or $A$ is an elementary abelian p-group for some prime $p$.

Proof: Let $T$ denote the torsion subgroup of $A$; then $T$ is normal in $G$ and so $T=1$ or $T=A$ and we may assume that $A$ is torsion and therefore a $p$-group for some prime $p$. Certainly $A$ is not minimal normal in $G$ and so $A$ contains a proper $G$-invariant subgroup $C$ of finite index. By local nilpotency $C$ may be chosen so that $|A / C|=p$. Let $B$ denote the intersection of all subgroups of index $p$ in $A$, so that $B$ is normal in $G$. If $B=1$ the result follows, so we shall assume for a contradiction that $A / B$ is finite and hence that $A=K B$ for some finite subgroup $K$. Now let $F$ be some finite subgroup of $A$. There exists a $G$-invariant subgroup $E$ of finite index in $A$ such that $F \cap E=1$. Let $L / E$ be the Frattini subgroup of $A / E$; thus $B \leq L$ and we have $A / E=K L / E$, which 
implies that $A / E=K E / E \cong K / K \cap E$ (by the usual property of the Frattini subgroup). But then the rank of $F$ is at most that of $K$, and the fact that $F$ was arbitrary tells us that $A$ has finite rank and is therefore Chernikov (see, for example, Corollary 2 of [10, Theorem 6.36]). But $A$ is residually finite and we obtain the contradiction that $A$ is finite.

With the hypotheses of the above lemma, if $A$ is torsion-free then it is in fact central in $G$ and therefore cyclic. This result is not essential for the proofs of the theorems but, apart from the fact that it allows us to establish a "just-infinite version" of Lemma 2.4 without the hypothesis that $G$ be a Baer group, it appears to be of interest in its own right. It is no further trouble to establish a somewhat stronger result, namely the following, which may indeed be well known.

Theorem 2.7. Let $G$ be a locally nilpotent group, $N$ a normal torsionfree subgroup of $G$, and supose that $N / M$ is periodic for all nontrivial $G$-invariant subgroups $M$ of $N$. Then $N$ is central in $G$ and hence of rank (at most) one.

Proof: Suppose that $N$ is not central, let $a \in N, g \in G$ with $[a, g] \neq 1$ and write $c=[a, g]$. By hypothesis $N /\langle c\rangle^{G}$ is periodic; in particular $\exists n \in \mathbb{N}$ such that $a^{n} \in\langle c\rangle^{G}$ and hence $a^{n} \in\langle c\rangle^{F}$ for some finitely generated subgroup $F$ of $G$. Let $H=\langle a, g, F\rangle, A=\langle a\rangle^{H}$, so that $c \in A$. Since $H$ is nilpotent we have $\left[A,{ }_{r} H\right]=1$ for some $r \in \mathbb{N}$. Now $c \in[A, H]$, a normal subgroup of $H$, and so $a^{n} \in[A, H]$. Since $A=\langle a\rangle[A, H]$ it follows that $A^{n} \leq[A, H]$. For each $i \geq 0$ write $A_{i}=\left[A,{ }_{i} H\right]$, and suppose that $A_{i}^{n} \leq A_{i+1}$ for some $i$. Then $A_{i+1}^{n}=\left[A_{i}, H\right]^{n}$ and, modulo $A_{i+2}$, we have $\left[A_{i}, H\right]$ central in $H$ and generated by elements $[x, y]$, where $x \in A_{i}$, $y \in H$, and so $\left(\bmod A_{i+2}\right)\left[A_{i}, H\right]^{n}$ is generated by the $n$th powers of such commutators. But $[x, y]^{n} \equiv\left[x^{n}, y\right] \bmod A_{i+2}$, by centrality, and we deduce that $A_{i+1}^{n} \leq\left[A_{i}^{n}, H\right] A_{i+2}=A_{i+2}$. By induction, therefore, $A^{n^{r}} \leq A_{r}=1$. But $A \leq N$ and $N$ is torsion-free and so we have $A=1$ and hence the contradiction $[a, g]=1$. Thus $N$ is central. If $z$ is a nontrivial element of $N$ then $\langle z\rangle$ is normal in $G$ and therefore $N /\langle z\rangle$ is periodic. The result follows.

Corollary 2.8. Let $G$ be a locally nilpotent group, $N$ a $G$-just infinite subgroup of $G$. If $N$ is torsion-free then $N$ is central and cyclic.

Proof: By Theorem $2.7 N$ is central. If $z$ is a nontrivial element of $N$ then $\langle z\rangle \triangleleft G$ and $N /\langle z\rangle$ is finite, so $N$ is cyclic. 
The following result may be compared with Lemma 2.3, where the hypothesis that $G$ be Baer could not be replaced by that of local nilpotency, as shown by the example $G=A]\langle g\rangle$ where $A \cong C_{p^{\infty}}$ and $g \in$ Aut $A$ is determined by $a^{g}=a^{p+1}$ for all $a \in A$.

Lemma 2.9. Let $G$ be a locally nilpotent group, $A$ a $G$-just infinite subgroup of $G$, and suppose that $G / C_{G}(A)$ is hypercentral. Then $A \leq Z(G)$. In particular, $A$ is infinite cyclic.

Proof: By Lemma 2.6 and Corollary 2.8 we need only dispose of the case where $A$ is assumed to be an elementary abelian $p$-group. Let $z C_{G}(A)$ be a nontrivial element of the centre of $G / C_{G}(A)$ and choose a nontrivial element $d$ of $A$ such that $[d, z]=1$ (such exists by local nilpotency). Let $g \in G$; by the choice of $z$ we have $1=[d, z]^{g}=\left[d^{g}, z\right]$ and so $z$ centralises $\langle d\rangle^{G}$. Now $A /\langle d\rangle^{G}$ is finite and the map $a \rightarrow[a, z]$ for all $a \in A$ is a homomorphism whose kernel contains $\langle d\rangle^{G}$, hence $[A, z]$ is finite (and $G$-invariant) and therefore trivial. This yields the contradiction that $z \in C_{G}(A)$ and thus establishes the lemma.

We have been unable to decide whether the hypothesis of solubility is necessary in the following.

Lemma 2.10. Let $G$ be a locally nilpotent group, $A$ a normal soluble subgroup of $G$ such that $G / C_{G}(A)$ is hypercentral. Suppose that $A$ satisfies max-G. Then $A$ is finitely generated and $A \leq Z_{n}(G)$ for some positive integer $n$.

Proof: An easy induction allows us to assume that $A$ is abelian. Let $T$ denote the torsion subgroup of $A$ and suppose first that $T$ is infinite. By Lemma 2.5, $T$ contains a $G$-invariant subgroup $U$ such that $T / U$ is $G$-just infinite; Lemma 2.9 now gives a contradiction. Thus $T$ is finite and, factoring, we may assume that $A$ is torsion-free. Again by Lemma 2.5 , there is a $G$-invariant subgroup $A_{1}$ of $A$ with $A / A_{1} G$-just infinite and hence, by Lemma 2.9 , infinite cyclic. If $A$ is not finitely generated then we obtain easily an infinite descending chain of $G$-invariant subgroups $A_{i}$ with $A_{i} / A_{i-1}$ infinite cyclic for each $i \geq 1$ (with $A_{0}=A$ ). Clearly $A / A_{i}$ is a free abelian group of rank exactly $i$ for each $i$. Now fix a prime $p$ and consider $A / A^{p}$; since this is a torsion group the previous argument shows that $A / A^{p}$ is finite of order $p^{r}$, say. But $A / A_{r+1}$ has a finite image of exponent $p$ and order $p^{r+1}$, a contradiction that shows that $A$ is finitely generated. We may now apply Lemma 6.37 of [10] to deduce that $A \leq Z_{n}(G)$ for some finite $n$, thus concluding the proof.

We are now ready to establish the final result of this section. 
Proposition 2.11. Let $G$ be a Baer group, $A$ a normal subgroup of $G$ such that $G / C_{G}(A)$ is hypercentral. If $A$ is $G$-minimax then $A$ is minimax and $A \leq Z_{n}(G)$ for some positive integer $n$.

Proof: By definition $A$ is soluble and, by induction on the length of an appropriate series, we may assume that $A$ is abelian and satisfies either $\max -G$ or min- $G$. Lemmas 2.4 and 2.10 now give the result.

\section{Conclusion}

Our main objective now is to prove Theorem $\mathrm{C}$, since Theorems A and $\mathrm{B}$ are easy consequences. The work of the previous section allows us to establish the following key result without difficulty.

Proposition 3.1. Let $G$ be a soluble Baer group and suppose that, for each element $x$ of $G,\langle x\rangle^{G}$ has a finite $G$-invariant series with abelian factors that are either torsion-free of finite rank or $G$-minimax. Then $G$ is hypercentral, with hypercentral length at most $\omega$.

Proof: Let $G / N$ be a hypercentral image of $G, U / V$ a $G$-invariant section of $N$. If $U / V$ is torsion-free of $\operatorname{rank} r$ then $\left[U{ }_{r} G\right] \leq V$ by Lemma 6.37 of [10], while if $U / V$ is $G$-minimax then, applying Proposition 2.11 to the group $G / N^{\prime}$, we see that there is an integer $n$ such that $\left[U N^{\prime},{ }_{n} G\right] \leq V N^{\prime}$. Now let $a \in N, D=\langle a\rangle^{G}$. The given hypotheses, together with the above argument, imply that $\exists m \in \mathbb{N}$ such that $\left[D N^{\prime},{ }_{m} G\right] \leq N^{\prime}$, and it follows that $N / N^{\prime}$ is contained in the hypercentre of $G / N^{\prime}$ and hence that $G / N^{\prime}$ is hypercentral. Since $G / G^{\prime}$ is certainly hypercentral, an easy induction on the derived length shows that $G$ is hypercentral. Now let $x$ be an arbitrary element of $G, X=\langle x\rangle^{G}, U / V$ a $G$-invariant section of $X$. Again by Lemma 6.37 of [10] and Proposition 2.11 we have $\left[U,{ }_{r} G\right] \leq V$ for some integer $r$, so that $X \leq Z_{m}(G)$ for some integer $m$. Since $x$ was arbitrary we have $G=Z_{\omega}(G)$ as required.

Our final prerequisite is a result that is probably well known. For the basic properties of isolators in locally nilpotent groups the reader is referred to $[\mathbf{3}]$.

Lemma 3.2. Let $G$ be a countable locally nilpotent group. Then there exists a torsion-free subgroup $K$ of $G$ whose isolator in $G$ is $G$. 
Proof: Write $G=\bigcup_{i=1}^{\infty} F_{i}$ where each $F_{i}$ is finitely generated and $F_{i} \leq$ $F_{i+1}$ for all $i$. Since the torsion subgroup of $F_{1}$ is finite there is a positive integer $n_{1}$ such that $K_{1}=: F_{1}^{n_{1}}$ is torsion-free; clearly $\left|F_{1}: K_{1}\right|$ is finite. Suppose that for some $i \geq 1$ we have a torsion-free subgroup $K_{i}$ of finite index in $F_{i}$ and let $K_{i+1}$ be a torsion-free subgroup of $F_{i+1}$ maximal with respect to containing $K_{i}$.

Claim. $\left|F_{i+1}: K_{i+1}\right|$ is finite. Supposing this false, write $F=F_{i+1}$, $H=K_{i+1}, I=I_{F}(H)$ (the isolator of $H$ in $F$ ). Then $|I: H|$ is finite and $\exists n>0$ with $I^{n} \leq H$. By hypothesis $I \leq F$ and so (by nilpotency) $I<N_{F}(I)$, hence $\exists g \in F \backslash I$ such that $I \triangleleft\langle I, g\rangle$. Clearly $g$ has infinite order module $I$. Since $I / I^{n}$ is finite $\exists k>0$ such that $\left[I,\left\langle g^{k}\right\rangle\right] \leq I^{n}$; but then $\left[H,\left\langle g^{k}\right\rangle\right] \leq I^{n} \leq H$ and $H$ is normal in $\left\langle H, g^{k}\right\rangle$. Since $\left\langle H, g^{k}\right\rangle / H$ is torsion-free we have a contradiction that establishes the claim.

Inductively, therefore, we may construct a chain $K_{1} \leq K_{2} \leq \cdots$ such that each $K_{i}$ is a torsion-free subgroup of finite index in $F_{i}$. Set $K=\bigcup_{i=1}^{\infty} K_{i}$; clearly $K$ satisfies the desired condition, and the lemma is proved.

Proof of Theorem $C$ : Let $G$ be as stated. By a result of Möhres $[\mathbf{9}] G$ is soluble and, by Proposition 3.1, $G$ is hypercentral of length at most $\omega$. Let $g \in G, D=\langle g\rangle^{G}$. Applying Proposition 2.11 we see that $G$-minimax sections of $D$ are in fact minimax and hence that $D$ has finite rank. Whereas the original hypothesis on normal closures is not in general inherited by subgroups and quotients of $G$, the hypothesis that each $\langle x\rangle^{G}$ have finite rank certainly is, and we now show that this condition is sufficient to ensure the nilpotency of the $\omega$-hypercentral group $G$.

By induction on the derived length of $G$ we may assume that $G^{\prime}$ is nilpotent. If $G / G^{\prime \prime}$ is nilpotent then so is $G[\mathbf{2}$, Theorem 7] and so we may factor and assume that $G$ is metabelian. We may also assume that $G$ is countable. Let $A$ be a normal abelian subgroup of $G$ with $G / A$ abelian. By Lemma 3.2 there is a torsion-free subgroup $K$ of $G$ with $I_{G}(K)=G$. Since $K$ is hypercentral of length at most $\omega$ it is nilpotent [14] and so $K A$, as a product of a normal and a subnormal nilpotent subgroup, is also nilpotent (see, for example, Proposition 3.3 .12 of $[7]$ ). If $G /(K A)^{\prime}$ is nilpotent then so is $G$; factoring if necessary we may therefore assume that $G / A$ is periodic. Now let $T$ be the torsion subgroup of $G$. By [8], $T$ is nilpotent and therefore so is $T A$. Since $(T A)^{\prime} \leq T$, the torsion subgroup of $G /(T A)^{\prime}$ is $T /(T A)^{\prime}$; factoring once more we may assume 
that $T \leq A$. Next, $G / T$ is torsion-free and abelian-by-periodic and hence abelian [10, Lemma 6.33]. Let $U$ be the divisible component of $T, C=C_{G}(U)$. Then $A \leq C$ and $G / C$ is periodic and, arguing as in the proof of Lemma 3.13 of $[\mathbf{1 0}]$, we deduce that $G / C$ is trivial. Then $U \leq Z(G)$ and we may (finally) assume that $U=1$ and hence that $T$ is reduced.

Let $x \in G$ and write $X=\langle x\rangle^{G}, Y=X \cap T$. Each $p$-component of $Y$ is Chernikov and reduced and therefore finite. Let $N$ be a $G$-invariant subgroup of finite index in $Y, E=C_{G}(Y / N)$, so that $G / E$ is finite. Since $G^{\prime} \leq T$ we see that $G$ centralises $X / Y$, and hence that $[X, E, E] \leq N$. By the Three Subgroup Lemma [10, Lemma 2.13], therefore, $\left[X, E^{\prime}\right] \leq N$. Now consider the group $G / E^{\prime}$, which is abelian-by-finite. Every abelian group $J$ is residually of rank 1 - this is well known and may be seen by noting that, for every nontrivial element $j$ of $J$, if $M$ is maximal with respect to not containing $j$ then $J / M$ is locally cyclic. It follows that $G / E^{\prime}$ is residually of finite rank. Now from the structure of $Y$ we have that the intersection of all $N$ defined as above is trivial and hence that the intersection $V_{x}$ of all the corresponding $E^{\prime}$ centralises $X$. But $G / V_{x}$ is also residually of finite rank and, further, so is $G / W$, where $W$ is the intersection of all $V_{x}$ obtained as $x$ runs through the set $G$. By Theorem 2 of $[\mathbf{1 3}] G / W$ is nilpotent. But $W \leq Z(G)$ and so $G$ is nilpotent and the proof of Theorem $\mathrm{C}$ is complete.

Theorem B is an immediate consequence of Theorem C, as is part (i) of Theorem A. Part (ii) follows from Proposition 2.11, and Theorem A is therefore proved.

Finally, we recall that paper [13] was concerned with establishing the nilpotency of $\omega$-hypercentral groups with all subgroups subnormal that have, in addition, certain rank restrictions on their structure. It does not appear to be known whether every $\omega$-hypercentral group with all subgroups subnormal is nilpotent (the examples in [12] having length $\omega+1$ ) and so it is perhaps worth recording the following result, which is what much of the proof of Theorem $\mathrm{C}$ was concerned with establishing.

Theorem 3.3. Let $G$ be a group with all subgroups subnormal and suppose that $G$ is hypercentral of length at most $\omega$. If $\langle x\rangle^{G}$ has finite rank for all $x$ in $G$ then $G$ is nilpotent.

\section{References}

1. L. FuCHS, "Infinite abelian groups," vol. 1, Academic Press, New York, 1970. 
2. P. HAll, Some sufficient conditions for a group to be nilpotent, Illinois J. Math. 2 (1958), 787-801.

3. P. Hall, The Edmonton Notes on nilpotent groups, QMC Notes, London (1969).

4. L. S. Kazarin and L. A. Kurdachenko, Finiteness conditions and factorizations in infinite groups, Russian Math. Surveys $\mathbf{4 7}$ (1992), 81-126.

5. L. A. Kurdachenko, A. V. Tushev and D. I. Zaitsev, Modules over nilpotent groups of finite rank, Algebra $i$ Logika 24 (1985), $631-666$.

6. L. A. KurdachenKo, On groups with minimax conjugacy classes in Infinite groups and the adjoining algebraic structures, Kiev (1990), 160-177.

7. J. C. Lennox And S. E. Stonehewer, "Subnormal subgroups of groups," Clarendon Press, Oxford, 1987.

8. W. MöHREs, Hyperzentrale Torsiongruppen, deren Untergruppen alle subnormal sind, Illinois J. Math. 35 (1991), 145-157.

9. W. MöHres, Auflösbarkeit von Gruppen, deren Untergruppen alle subnormal sind, Arch. Math. 54 (1990), 232-235.

10. D. J. S. Robinson, "Finiteness conditions and generalized soluble groups," 2 vols., Springer, New York-Heidelberg-Berlin, 1972.

11. D. J. S. Robinson And J. S. Wilson, Soluble groups with many polycyclic quotients, Proc. London Math. Soc. 48 (1984), 193-229.

12. H. Smith, Hypercentral groups with all subgroups subnormal, Bull. London Math. Soc. 15 (1983), 229-234.

13. H. Sмiтh, Hypercentral groups with all subgroups subnormal II, Bull. London Math. Soc. 18 (1986), 343-348.

14. H. Sмiтh, On torsion-free hypercentral groups with all subgroups subnormal, Glasgow Math. J. 31 (1989), 193-194.

15. H. Smith, A note on locally soluble, normal closures of cyclic subgroups, Glasgow Math. J. 36 (1994), 33-36. 
16. D. I. ZAITSEV, Infinitely irreducible normal subgroups in Structures of groups and properties of their subgroups, Kiev Inst. Math (1978), $17-38$.

Leonid A. Kurdachenko:

University of Dnepropetrovsk

Dnepropetrovsk 94

320094 UKRAINE
Howard Smith:

Bucknell University

Lewisburg PA 17837

U.S.A.

Rebut el 3 de setembre de 1997 